\title{
ICU Clinicians Underestimate Breathing Discomfort in Ventilated Subjects
}

\author{
Andrew P Binks PhD, Steven Desjardin RRT, and Richard Riker MD
}

\begin{abstract}
BACKGROUND: Breathing discomfort (dyspnea) during mechanical ventilation in the ICU may contribute to patient distress and complicate care. Assessment of nonverbal cues may allow caregivers to estimate patient breathing discomfort. This study assesses the accuracy of those caregiver estimates. METHODS: Thirty subjects were identified from ventilated, hemodynamically stable patients in the special care unit of Maine Medical Center. Those with impaired neurological function or too unstable to waken were excluded. Subjects provided a subjective score of breathing discomfort (0-10 using a modified Borg scale) during daily wake-up from sedation (sedationagitation score of 3 or 4). Clinicians (physicians, respiratory therapists, and nurses) then provided a blinded estimate of subject breathing discomfort (0-10) through observation of the subject and inspection of ventilator parameters alone. Subject scores and caregiver estimates were compared. RESULTS: All subjects reported breathing discomfort with median score (interquartile range) of 4 (3-4). Caregiver estimates of breathing discomfort were significantly lower than subject scores (2 $[0-3]$ ), and the discrepancy was seen in all professions (physicians 1 point lower $[0-2], P=.02$; respiratory therapists 1 point lower $[0-2], P=.01$; nurses 2 points lower [1-3], $P<.001)$. There was a positive correlation between subject breathing discomfort and degree of underestimation (ie, the degree of underestimation increased as the subject scores rose). The 3 most commonly used cues were subjects' facial expression, use of accessory muscles, and nasal flaring. CONCLUSIONS: Significant breathing discomfort is prevalent in mechanically ventilated ICU patients and is underestimated by caregivers, regardless of profession. The increasing disparity in caregiver estimate as breathing discomfort rises may expose patients to levels of dyspnea that promote anxiety and fear. This study demonstrates the need for further development and standardization of methods to assess dyspnea in nonverbal patients. Key words: dyspnea; mechanical ventilation; sedation; patient acuity; methods; intensive care. [Respir Care 2017;62(2):150-155. () 2017 Daedalus Enterprises]
\end{abstract}

\section{Introduction}

Breathing discomfort is among the stressful factors experienced and remembered by the majority of mechani-

Dr Binks is affiliated with the University of South Carolina School of Medicine, Greenville, South Carolina. Mr Desjardin and Dr Riker are affiliated with the Maine Medical Center, Portland, Maine. Dr Riker is also affiliated with the Tufts University School of Medicine, Boston, Massachusetts.

This study was supported by a Patient and Population Orientated Research Award from the University of New England. The authors have disclosed no conflicts of interest.

Dr Riker presented a version of this report at the Society for Critical Care Medicine's 43rd Critical Care Congress, held January 9-13, 2014, in San Francisco, California. cally ventilated ICU patients. ${ }^{1}$ Most of these patients report the discomfort as "air hunger," which carries a strong affective component ${ }^{2}$ that manifests as anxiety and fear. Despite the call for more patient-centered mechanical ventilation being made 15 years ago in this journal, ${ }^{3}$ little attention has been paid to the detection, management, or long-term consequences of breathing discomfort of ventilated patients.

Although numerous factors contribute to anxiety in the ICU (eg, pain, delirium, loss of communication), air hun-

\footnotetext{
Correspondence: Andrew P Binks PhD, University of South Carolina School of Medicine, Greenville, 701 Grove Road, Greenville, SC 29605. E-mail: binks@greenvillemed.sc.edu.
}

DOI: $10.4187 /$ respcare. 04927 
ger is arguably our most urgent homeostatic warning signal. As such, it is unpleasant and brings immediate suffering to the patient, whereas the anxiety it generates produces long-lasting sequelae. ${ }^{4}$ Nearly 1 in 5 ICU survivors suffer from post-traumatic stress disorder, ${ }^{5}$ and its incidence has been associated with mechanical ventilation $^{6,7}$ and the recall of dyspnea. ${ }^{8}$

\section{See the Related Editorial on Page 250}

It should not be surprising that air hunger is prevalent in the ICU environment because it is the cardinal symptom of cardiovascular and bronchopulmonary disorders, and current practice favors protective ventilation protocols that restrict tidal volume that exacerbate air hunger. ${ }^{4,9}$ The common response to apparent breathing discomfort is to sedate the patient, but conversely, there are now demands to reduce or lighten sedation due to its deleterious consequences.

If significant respiratory discomfort and its sequelae are to be addressed, improved detection and alternative management protocols with less reliance on sedation must be developed. This single-center study assesses how well a nonverbal subject's breathing discomfort is assessed by ICU clinicians.

\section{Methods}

The institutional review board approved this study and waived the requirement for informed consent. We approached each patient and family regarding this observational study and did not proceed unless they agreed to take part. Recruitment of ICU clinicians was also approved by the institutional review board, and again the requirement for informed consent was waived.

\section{Patient Population}

The subjects $(N=30)$ were identified by a member of the research team from a 32-bed multidisciplinary special care unit. All subjects had been intubated or had tracheostomies for at least $48 \mathrm{~h}$ before selection, and all were hemodynamically stable. Patients who were receiving neuromuscular blockade, had severe neurologic impairments, or were deemed too unstable to waken were excluded. The identified subject's attending physician was contacted and had to approve approaching the patient and family for enrollment. The cause of respiratory failure, number of days on the ventilator, and prescribed sedatives and sedation level of the subjects were recorded.

\section{ICU Clinicians}

The subject's nurse (RN), respiratory therapist (RT), and attending physician (MD) were invited to participate

\section{QUICK LOOK}

\section{Current knowledge}

Breathing discomfort is a common symptom suffered by mechanically ventilated ICU patients. Most patients report their discomfort as "air hunger" that produces fear and anxiety and can lead to long-term deleterious sequalae. Despite this, little attention has been paid to the detection and management of breathing discomfort in mechanically ventilated patients.

\section{What this paper contributes to our knowledge}

Comparison of subject scores and clinicians' estimates shows that clinicians persistently underestimated the degree of breathing discomfort suffered by ventilated subjects in the ICU. More concerning is that the discrepancy between subject score and clinician estimate increased with greater degrees of subject discomfort.

in the study during the selection process. The clinicians were told that the purpose of the study was to assess breathing discomfort and that any responses or information they gave would be anonymous.

\section{Protocol}

The 15-30-min assessment of breathing discomfort was performed when the following criteria were met: (1) subject was awake with a sedation-agitation scale ${ }^{10}$ score of 3-4 (ie, able to follow simple commands); (2) there were no plans for the subject to leave the ICU, change the mode of ventilator support, or undergo any procedure in the next hour; and (3) a nurse, RT, and physician were available to evaluate the subject. The protocol began with a research team member recording the ventilator settings and asking the subject to report their breathing discomfort (the same team member did not provide a breathing discomfort estimate). The subject score was made using a modified Borg scale. ${ }^{11}$ The scale was printed on paper so that a nonverbal subject could finger-point to indicate his or her score. Instructions were kept simple and were limited to "Please let us know how uncomfortable your breathing is by pointing to a number on this scale, where 0 is no discomfort and 10 is extremely uncomfortable." A blinded independent estimate of subject breathing discomfort was then performed by the RN, RT, and MD within 15 min of the subject's score being recorded. Clinicians were told that they could use any parameters or observations to make their estimation, and they were asked what cues they used afterward. They were not allowed to ask the subject about his or her degree of breathing discomfort. 


\section{Underestimation of BREATHING Discomfort IN THE ICU}

\section{Statistical Analysis}

To determine whether patient-related parameters might have influenced breathing discomfort and clinician assessment, the subjects' breathing discomfort scores were compared with diagnosis (cardiopulmonary or other) and the level of alertness (sedation-agitation scale score $=3$ or 4 ) using a Mann-Whitney U test (2-tailed, significance level of $P<.05)$. The degree of correlation between subject score of breathing discomfort and each clinical discipline's (MD, RT, and RN) estimate was determined using Spearman rank correlation coefficients. The subject's score was also compared with MD, RT, and RN estimates using a Mann-Whitney U test (2-tailed, significance level of $P<.05)$ to assess whether any score-estimate discrepancy was significant. Spearman rank correlation coefficients were used to determine whether the degree of subject breathing discomfort and the score-estimate discrepancy were related. Descriptive statistics of non-continuous data are reported as median (interquartile range), and continuous data are reported as mean $\pm \mathrm{SD}$.

\section{Results}

\section{Subject Population}

Subject details, including reasons for ventilation and sedation-agitation scale score at time of providing a breathing discomfort score, are listed in Table 1 along with the associated breathing discomfort score. The median subject breathing discomfort score was 4 (4-7), with all subjects reporting some degree of discomfort. There was no significant difference in the breathing discomfort reported by subjects primarily ventilated for cardiopulmonary issues and those ventilated for other issues (median 6 [4-7] vs 4 [4-5], respectively, $P=.56$ ); nor was there any effect of subject sex $(P=.26)$. All subjects had a sedationagitation scale score of 3 or 4 at the time of breathing discomfort scoring, and there was no effect of sedationagitation scale score on reported breathing discomfort (median 4 [4-5] vs 5 [4-7], respectively, $P=.37$ ).

\section{Comparison of Subject Breathing Discomfort Scores and Clinician Estimates}

No clinician who was asked to participate in the study refused to do so. Twenty-one subjects were assessed by all 3 of each subject's clinicians (MD, RT, and RN); only the $\mathrm{RN}$ and RT of the remaining 9 subjects gave estimates (no MD was immediately available). There was a significant positive correlation between subject breathing discomfort score and the estimates given by respiratory therapists $(\mathrm{r}=0.467, P=.01)$, but the estimates by physicians $(\mathrm{r}=0.382, P=.09)$ and nurses $(\mathrm{r}=0.260, P=.17) \mathrm{did}$
Table 1. Subject and Ventilator Parameters at the Time of Making a Breathing Discomfort Score

\begin{tabular}{|c|c|}
\hline Subject Parameters & $\begin{array}{c}\text { Breathing Discomfort, } \\
\text { Median (IQR) }\end{array}$ \\
\hline \multicolumn{2}{|l|}{ Sex } \\
\hline Female $(n=8)$ & $6(4-7)$ \\
\hline Male $(n=22)$ & $4(3-5)$ \\
\hline \multicolumn{2}{|c|}{ Primary reason for mechanical ventilation } \\
\hline Airway protection $(n=2)$ & 4 and 3 \\
\hline $\mathrm{CHF}(n=3)$ & $4(3-5)$ \\
\hline $\operatorname{COPD}(n=2)$ & 10 and 4 \\
\hline Pneumonia $(n=4)$ & $7(7-8)$ \\
\hline Post-operative $(n=7)$ & $4(3-6)$ \\
\hline Trauma $(n=11)$ & $4(4-5)$ \\
\hline Asthma $(n=1)$ & 7 \\
\hline \multicolumn{2}{|l|}{ Prior sedation } \\
\hline Fentanyl $(n=14)$ & $4(3-6)$ \\
\hline None $(n=16)$ & $5(4-7)$ \\
\hline \multicolumn{2}{|l|}{ Sedation-agitation score } \\
\hline $3(n=13)$ & $4(4-5)$ \\
\hline $4(n=17)$ & $5(4-7)$ \\
\hline Ventilator Parameters & Mean $\pm \mathrm{SD}$ (range) \\
\hline \multicolumn{2}{|l|}{ Ventilator mode } \\
\hline \multicolumn{2}{|l|}{$\operatorname{CSV}(n=15)$} \\
\hline \multicolumn{2}{|l|}{$\operatorname{VC-CMV~}(n=11)$} \\
\hline \multicolumn{2}{|l|}{$\operatorname{APRV}(n=3)$} \\
\hline \multicolumn{2}{|l|}{ VC-IMV $(n=1)$} \\
\hline \multicolumn{2}{|l|}{ Other ventilator parameters } \\
\hline Ventilator duration, $\mathrm{d}$ & $5.7 \pm 4.2(1-16)$ \\
\hline $\mathrm{F}_{\mathrm{IO}_{2}}, \%$ predicted & $39.5 \pm 5.0 \%(25-50)$ \\
\hline Mean tidal volume, $\mathrm{L}$ & $432 \pm 113 \mathrm{ml}(200-650)$ \\
\hline Breathing frequency, breaths/min & $19.5 \pm 5.9(10-36)$ \\
\hline $\mathrm{PEEP}, \mathrm{cm} \mathrm{H}_{2} \mathrm{O}$ & $5.6 \pm 1.7(0-8)$ \\
\hline $\begin{array}{l}\text { IQR = interquartile range } \\
\text { CSV = continuous spontaneous ventilation } \\
\text { VC-CMV = volume control continuous mandato } \\
\text { APRV = airway pressure release ventilation } \\
\text { VC-IMV = volume control intermittent ventilati }\end{array}$ & \\
\hline
\end{tabular}

not reach statistical significance. Overall, the clinicians underestimated subjects' breathing discomfort by a median (interquartile range) of 2 scale points $(0-3)$. The underestimation was significant for all disciplines, with physicians and respiratory therapists both underestimating patient breathing discomfort by a median of 1 scale point (interquartile range $0-2, P=.02$, and interquartile range $0-2, P=.01$, respectively) and nurses by 2 scale points (interquartile range $1-3, P<.001$ ). The underestimation tended to become larger when subject breathing discomfort scores were higher (illustrated by the linear regression lines diverging from the line of unity in Fig. 1). This positive correlation between subject breathing discomfort and the discrepancy with clinician estimate was significant 

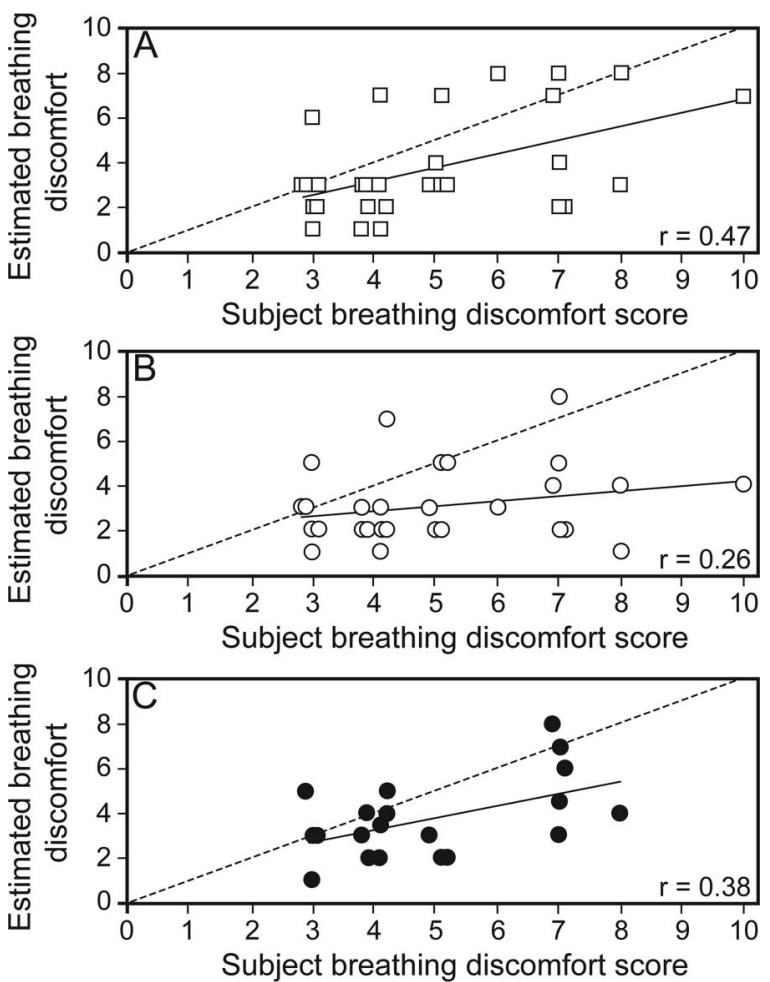

Fig. 1. Comparison of respiratory therapist (A), nurse (B), and physician (C) estimates of breathing discomfort and the breathing discomfort score of subjects. Dashed lines are the lines of unity (ie, where an estimate would equal a score). Solid regression lines and coefficients ( $r$ ) of subject breathing discomfort scores and each profession's estimates are shown. Some subjects' scores have been shifted by \pm 0.1 to prevent overlaying points obscuring each other (all subject scores were integers).

for RNs $(\mathrm{r}=-0.627, P<.001)$ but not RTs $(\mathrm{r}=-0.319$, $P=.09)$ or MDs ( $=0.002, P=.99)$.

The discrepancy between subject breathing discomfort score and clinician estimate was not affected by the primary reason for ventilation (ie, cardiopulmonary or other) (RNs, $P=.85$; RTs, $P=.83$; MDs, $P=.22$ ), suggesting that knowledge of the subject's condition did not lead to an expectation bias. Likewise, there was no effect of subject sex on the score-estimate discrepancy (RNs, $P=.66$; RTs, $P=.48$; MDs, $P=.97$ ).

\section{Cues Used by Clinicians to Determine an Estimate of Breathing Discomfort}

We did not have sufficient data to perform a formal analysis of the efficacy of the cues clinicians used to estimate breathing discomfort, but some trends were apparent. The number of cues used for a single estimate ranged between 1 and 7 (mode $=3$ ). The RTs tended to look for evidence of asynchrony in the ventilator flow patterns and backed this up with subject assessment (use of accessory muscles, restlessness, nasal flaring, and facial expression). The MDs typically started with subject assessment, particularly with facial expression but also nasal flaring, restlessness, and use of accessory muscles. RNs typically looked at heart rate and breathing frequency with the more experienced being able to recognize that the subject was "fighting" the ventilator from observation of the subject.

\section{Discussion}

Although this is only a single-center study, it has 2 important findings. First, ICU subjects had significant breathing discomfort (confirming earlier reports ${ }^{1,12,13}$ ), and clinicians underestimated this discomfort. The degree of underestimation, although relatively small on our scale (10-20\% of full scale), is at or above the threshold of clinical importance in terms of subject comfort (10\%). ${ }^{14}$ Second, our finding that the degree of underestimation of breathing discomfort increases at higher levels of subject discomfort is perhaps even more clinically pertinent. The ramification for underestimation is undertreatment, and undertreating breathing discomfort leads to short-term suffering and long-term sequelae. A recent study by Haugdahl et al ${ }^{15}$ showed that physicians and nurses underestimate patient breathlessness and feeling of security during spontaneous breathing trials; a poor appreciation of patient discomfort/distress may adversely affect the timeliness of the decision to wean the patient off of mechanical ventilation. This and our data assessing breathing discomfort during mechanical ventilation confirm the need to (1) improve breathing discomfort detection and (2) devise better management protocols that avoid continuous sedation.

The simplest initial approach to detecting discomfort is to ask the patient, a principle promoted by the American Pain Society since 1996 ("pain as the fifth vital sign"). But the nonverbal patient presents challenges that have only recently started to be addressed. Determining respiratory distress from facial expression (particularly that of fear), use of accessory muscles, nasal flaring, and physiological signs (heart rate and breathing frequency) has been described ${ }^{16}$ and validated ${ }^{17}$ in Campbell's Respiratory Distress Observation Scale. The Respiratory Distress Observation Scale scores correlate to patient scores $(r=0.398)$ to a similar degree as our ICU clinicians' estimates of breathing discomfort over a similar range of dyspnea, and the Respiratory Distress Observation Scale has recently been used with ventilated patients. ${ }^{18}$ But Campbell's validation study ${ }^{18}$ does not report any consistent underestimation as we have observed in clinicians using their own criteria (although many overlapped with Respiratory Distress Observation Scale criteria). Also, dyspnea measurement was not a routine part of clinical care at our study site but is addressed on an ad hoc basis when signs of breathing discomfort are apparent. This informal approach may 
explain the difference from the more rigorous Respiratory Distress Observation Scale criteria. Use of accessory muscles and facial expression (the second and third best dyspnea correlates in the Respiratory Distress Observation Scale) were the most regularly used by our clinicians. Although an area for more thorough study, it is worth noting that RNs who spend the greatest time with patients produced the least accurate breathing discomfort estimates (also observed by Haugdahl et $\mathrm{al}^{15}$ ), perhaps because of their reliance on physiological signs. The most accurate estimates tended to include interpretation of ventilator flow signals, but these were less frequently used.

Signs of patient-ventilator asynchrony ("fighting" or "bucking" the ventilator) have been studied, but the focus has been on the physiological rather than psychological correlates of asynchrony ${ }^{19-21}$ or the effects of different ventilator modes. ${ }^{11}$ Although inspection of flow and pressure signals is an established clinical technique, it remains more an art than an empirical science, and more descriptive studies might validate, improve, or even automate this process. Similarly, other quantifiable signs of overt patient efforts to breathe, such as respiratory muscle electromyogram or transdiaphragmatic pressures, ${ }^{22}$ might also be considered but would have to be viable in a busy clinical environment.

Although quantifying pain has become standard practice, its treatment as the fifth vital sign has done little to improve pain management. ${ }^{23}$ Similarly, the response to emerging reports of breathing discomfort must include studies and implementation of viable management plans. Although our understanding of dyspnea languishes decades behind our understanding of pain, viable options to improve the comfort of ventilated patients exist. Changing ventilator settings can provide immediate relief. Raising the level of ventilator assistance provides more comfort. ${ }^{24,25}$ Relief is also afforded by increasing inspiratory flows, ${ }^{26}$ raising tidal volume, ${ }^{1,4}$ or increasing end-expiratory volume. ${ }^{27}$ Alternative methods would have to be used for patients where lung expansion is purposefully limited (eg, those with $\mathrm{ARDS}^{28}$ ). These alternatives might include increasing inspiratory resistance during early expiration, ${ }^{29}$ blowing air on the patient's face, ${ }^{30,31}$ or use of acupressure, ${ }^{32}$ or more selective use of sedatives to reduce duration of ventilation ${ }^{33}$ might be considered. Again, this is an area worthy of more investigation.

Although the consistent and significant trends of underestimating breathing discomfort might ameliorate concerns about our small sample size, a more robust data set would allow better interpretation of the efficacy of the signs and cues that clinicians used to estimate breathing discomfort. Likewise, our data are from a single institution, and practices and training may differ at other medical centers, which might result in different degrees of score-estimate discrepancy. Finally, our accepted standard of breathing discom- fort is qualitatively limited (ie, does not discriminate between types of breathing discomfort) and was provided by awake but recently sedated subjects. Given the importance of specific and real-time patient scores rather than retrospective and general patient reports, this problem would seem to be insurmountable. Also, there was the possibility that the subject's breathing discomfort changed in the time between giving their score and the observation by the clinicians ( $<15 \mathrm{~min}$ ), but, since the subjects were hemodynamically stable and the underestimation by clinicians was so consistent, we do not believe that this was a prevalent problem.

\section{Conclusions}

This study suggests that breathing discomfort is common and, like other symptoms, such as pain and the symptoms of asthma, is underestimated by clinical staff. ${ }^{34-36}$ The degree of underestimation was clinically relevant, ${ }^{14}$ consistently became greater at higher levels of subject discomfort (a phenomenon also seen with clinicians' estimates of pain ${ }^{34,37}$ ), and appeared unrelated to the amount of time spent with the subject. Although components of the Respiratory Distress Observation Scale have been adopted on an ad hoc basis by some clinicians, we advocate for the use of more robust breathing assessment in the ICU. Further study will allow us to better describe the problem of breathing discomfort during mechanical ventilation and refine areas for clinician training to improve its detection and ultimately reduce the incidence of this common symptom with long-lasting sequelae.

\section{ACKNOWLEDGMENTS}

We thank the staff of Maine Medical Center for thoughtful and committed participation in the study. We also thank Dawn Blackhurst PhD for invaluable help with the study's statistical analysis.

\section{REFERENCES}

1. Schmidt M, Demoule A, Polito A, Porchet R, Aboab J, Siami S, et al. Dyspnea in mechanically ventilated critically ill patients. Crit Care Med 2011;39(9):2059-2065.

2. Lansing RW, Gracely RH, Banzett RB. The multiple dimensions of dyspnea: review and hypotheses. Respir Physiol Neurobiol 2009; 167(1):53-60

3. Hansen-Flaschen JH. Dyspnea in the ventilated patient: a call for patient-centered mechanical ventilation. Respir Care 2000;45(12): 1460-1464, discussion 1464-1467.

4. Schmidt M, Banzett RB, Raux M, Morelot-Panzini C, Dangers L, Similowski T, et al. Unrecognized suffering in the ICU: addressing dyspnea in mechanically ventilated patients. Intensive Care Med 2014;40(1):1-10.

5. Ratzer M, Brink O, Knudsen L, Elklit A. Posttraumatic stress in intensive care unit survivors: a prospective study. Health Psychol Behav Med 2014;2(1):882-898. 


\section{Underestimation of BREATHING Discomfort IN THE ICU}

6. Caiuby AV, Andreoli PB, Andreoli SB. Post-traumatic stress disorder in intensive care unit patients. Rev Bras Ter Intensiva 2010; 22(1):77-84.

7. Cuthbertson BH, Hull A, Strachan M, Scott J. Post-traumatic stress disorder after critical illness requiring general intensive care. Intensive Care Med 2004;30(3):450-455.

8. Schelling G. Effects of stress hormones on traumatic memory formation and the development of posttraumatic stress disorder in critically ill patients. Neurobiol Learn Mem 2002;78(3):596-609.

9. Binks AP, Evans KC, Reed JD, Moosavi SH, Banzett RB. The time-course of cortico-limbic neural responses to air hunger. Respir Physiol Neurobiol 2014;204:78-85.

10. Riker RR, Picard JT, Fraser GL. Prospective evaluation of the sedation-agitation scale for adult critically ill patients. Crit Care Med 1999;27(7):1325-1329.

11. Powers J, Bennett SJ. Measurement of dyspnea in patients treated with mechanical ventilation. Am J Crit Care 1999;8(4):254-261.

12. Nelson JE, Meier DE, Oei EJ, Nierman DM, Senzel RS, Manfredi PL, et al. Self-reported symptom experience of critically ill cancer patients receiving intensive care. Crit Care Med 2001;29(2):277-282.

13. Puntillo KA, Arai S, Cohen NH, Gropper MA, Neuhaus J, Paul SM, Miaskowski C. Symptoms experienced by intensive care unit patients at high risk of dying. Crit Care Med 2010;38(11):2155-2160.

14. Johnson MJ, Bland JM, Oxberry SG, Abernethy AP, Currow DC. Clinically important differences in the intensity of chronic refractory breathlessness. J Pain Symptom Manage 2013;46(6):957-963.

15. Haugdahl HS, Storli SL, Meland B, Dybwik K, Romild U, Klepstad P. Underestimation of patient breathlessness by nurses and physicians during a spontaneous breathing trial. Am J Respir Crit Care Med 2015;192(12):1440-1448

16. Campbell ML. Respiratory distress: a model of responses and behaviors to an asphyxial threat for patients who are unable to selfreport. Heart Lung 2008;37(1):54-60.

17. Campbell ML. Psychometric testing of a respiratory distress observation scale. J Palliat Med 2008;11(1):44-50.

18. Campbell ML, Yarandi HN, Mendez M. A two-group trial of a terminal ventilator withdrawal algorithm: pilot testing. J Palliat Med 2015;18(9):781-785.

19. Nilsestuen JO, Hargett KD. Using ventilator graphics to identify patient-ventilator asynchrony. Respir Care 2005;50(2):202-234; discussion 232-234.

20. Branson RD, Blakeman TC, Robinson BR. Asynchrony and dyspnea. Respir Care 2013;58(6):973-989.

21. Kondili E, Akoumianaki E, Alexopoulou C, Georgopoulos D. Identifying and relieving asynchrony during mechanical ventilation. Expert Rev Respir Med 2009;3(3):231-243.

22. Hug F, Raux M, Morelot-Panzini C, Similowski T. Surface EMG to assess and quantify upper airway dilators activity during non-invasive ventilation. Respir Physiol Neurobiol 2011;178(2):341-345.
23. Mularski RA, White-Chu F, Overbay D, Miller L, Asch SM, Ganzini L. Measuring pain as the 5th vital sign does not improve quality of pain management. J Gen Intern Med 2006;21(6):607-612.

24. Leung P, Jubran A, Tobin MJ. Comparison of assisted ventilator modes on triggering, patient effort, and dyspnea. Am J Respir Crit Care Med 1997;155(6):1940-1948.

25. Mols G, von Ungern-Sternberg B, Rohr E, Haberthür C, Geiger K, Guttmann J. Respiratory comfort and breathing pattern during volume proportional assist ventilation and pressure support ventilation: a study on volunteers with artificially reduced compliance. Crit Care Med 2000;28(6):1940-1946.

26. Manning HL, Molinary EJ, Leiter JC. Effect of inspiratory flow rate on respiratory sensation and pattern of breathing. Am J Respir Crit Care Med 1995;151(3 Pt 1):751-757.

27. Vovk A, Binks AP. Raising end-expiratory volume relieves air hunger in mechanically ventilated healthy adults. J Appl Physiol 2007; 103(3):779-786.

28. The Acute Respiratory Distress Syndrome Network. Ventilation with lower tidal volumes as compared with traditional tidal volumes for acute lung injury and the acute respiratory distress syndrome. N Engl J Med 2000;342(18):1301-1308.

29. Wirth S, Best C, Spaeth J, Guttmann J, Schumann S. Flow controlled expiration is perceived as less uncomfortable than positive end expiratory pressure. Respir Physiol Neurobiol 2014;202:59-63.

30. Schwartzstein RM, Lahive K, Pope A, Weinberger SE, Weiss JW. Cold facial stimulation reduces breathlessness induced in normal subjects. Am Rev Respir Dis 1987;136(1):58-61.

31. Galbraith S, Fagan P, Perkins P, Lynch A, Booth S. Does the use of a handheld fan improve chronic dyspnea? A randomized, controlled, crossover trial. J Pain Symptom Manage 2010;39(5):831-838.

32. Tsay SL, Wang JC, Lin KC, Chung UL. Effects of acupressure therapy for patients having prolonged mechanical ventilation support. J Adv Nurs 2005;52(2):142-150.

33. Riker RR, Shehabi Y, Bokesch PM, Ceraso D, Wisemandle W, Koura F, et al. Dexmedetomidine vs midazolam for sedation of critically ill patients: a randomized trial. JAMA 2009;301(5):489499.

34. Mäntyselkä P, Kumpusalo E, Ahonen R, Takala J. Patients' versus general practitioners' assessments of pain intensity in primary care patients with non-cancer pain. Br J Gen Pract 2001;51(473):995-997.

35. Petersen MA, Larsen H, Pedersen L, Sonne N, Groenvold M. Assessing health-related quality of life in palliative care: comparing patient and physician assessments. Eur J Cancer 2006;42(8):11591166

36. König P, Rejent A. Subjective and objective means of assessing cystic fibrosis and asthma. Ann Allergy 1982;49(2):86-92.

37. Idvall E, Brudin L. Do health care professionals underestimate severe pain more often than mild pain? Statistical pitfalls using a data simulation model. J Eval Clin Pract 2005;11(5):438-443.

This article is approved for Continuing Respiratory Care Education credit. For information and to obtain your CRCE

(free to AARC members) visit

www.rcjournal.com

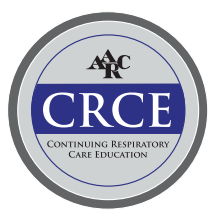

